15

\title{
Холловский микроскоп для измерения магнитных свойств пленок
}

\author{
() В.К. Игнатьев, А.А. Орлов Ф, С.В. Перченко, Д.А. Станкевич
}

Волгоградский государственный университет

`E-mail: orlwork@inbox.ru

Поступило в Редакцию 17 июля 2016 г.

В окончательной редакции 13 апреля 2017 г.

Описан магнитный измерительный микроскоп, обладающий пространственным разрешением определения координат токовых диполей $3 \mu \mathrm{m}$. В качестве первичного преобразователя используется пленочный датчик Холла с габаритами чувствительной зоны $250 \times 400 \mu \mathrm{m}$, размещенный на расстоянии $3 \mathrm{~mm}$ от плоскости сканирования. Сверхразрешение по сравнению с размерами датчика и расстоянием до объекта достигнуто путем использования априорной информации об объекте сканирования. Предложен способ калибровки магнитного микроскопа и измерения его метрологических характеристик. Сравнением случайной погрешности измерения координат токового диполя с пределом Рао-Крамера показана эффективность и состоятельность полученной оценки координат. Измерительный микроскоп может применяться при исследовании элементов функциональной электроники.

DOI: 10.21883/PJTF.2017.15.44864.16434

Одним из наиболее информативных методов анализа электромагнитных свойств материалов является сканирующая магнитная микроскопия. Разрешение получаемых магнитных топографий определяется расстоянием от датчика до поверхности исследуемого образца и размером чувствительной зоны датчика. В работе [1] достигнуто разрешение $\sim 0.85 \mu \mathrm{m}$ благодаря использованию микродатчика Холла 
с размером активной зоны $1 \times 1 \mu \mathrm{m}$ и расстоянием до поверхности объекта $13 \mu \mathrm{m}$. Недостатком микродатчика является малое отношение сигнал/шум даже при температуре $77 \mathrm{~K}$ [1]. В работах [2,3] для измерения используется стандартный датчик Холла с размерами активной зоны $450 \times 150 \mu \mathrm{m}$. После модификации удалось приблизить датчик к исследуемой поверхности на расстояние $\sim 10 \mu \mathrm{m}$ и добиться разрешения получаемых топографий $\sim 50 \mu \mathrm{m}$. Высокое разрешение в работах $[2,3]$ достигается применением уникальной техники физического эксперимента, обеспечивающей визуализацию магнитного состояния объекта исследования в области, линейные размеры которой в 30-100 раз превышают порог разрешения микроскопа и ограничены применяемой системой микроперемещений.

Для широкого применения магнитной микроскопии в таких областях технической физики, как лабораторные исследования и оперативный микроструктурный анализ материалов и изделий функциональной электроники, необходимо в установке на базе серийного оборудования сочетать высокую чувствительность с точностью измерения геометрических и магнитных параметров в большом динамическом диапазоне.

Задача нашей работы состоит в получении сверхразрешения магнитного микроскопа по сравнению с размерами датчика и расстоянием до объекта. Для этого используется параметрическая модель объекта сканирования. Точность измерения параметров обеспечивается периодической калибровкой микроскопа по ленточному токовому диполю.

Разработанный магнитный микроскоп представляет собой программно-аппаратный комплекс определения параметров токовых распределений заранее известной геометрии в тонком образце по измеряемой вне его топографии магнитного поля. Неизвестными параметрами являются координаты токового распределения и углы ориентации токового распределения относительно калибровочного диполя. Аппаратная часть состоит из холловского магнитометра [4] и системы позиционирования датчика.

Магнитометр на основе датчика Холла ПХЭ602117 (ООО „Сенсор“, Санкт-Петербург) с размером активной зоны $250 \times 400 \mu \mathrm{m} \mathrm{и}$ чувствительностью $1 \mathrm{~V} / \mathrm{T}$ при токе $100 \mathrm{~mA}$ имеет среднее квадратичное отклонение (CKO) значений отсчетов индукции магнитного поля порядка $0.1 \mu \mathrm{T}$. Систематическая погрешность преобразователей устраняется применением четырехтактного алгоритма измерения [5], при котором стабильный ток поочередно пропускается в разных направлениях через

Письма в ЖТФ, 2017, том 43, вып. 15 
токовые и потенциальные выводы датчика и измеряются напряжения с потенциальных и токовых контактов соответственно. Комбинация этих напряжений пропорциональна индукции внешнего магнитного поля, а поле самовоздействия токов, протекающих по датчику и его выводам, при этом вычитается. Коммутация выводов осуществляется с частотой $12 \mathrm{~Hz}$. В качестве измерительного каскада используется микросхема AD7190, имеющая коммутатор входных каскадов, малошумящий предварительный усилитель и аналого-цифровой преобразователь с разрядностью 24 бита.

Система перемещения реализована на базе программно-управляемого станка ЕР2006Н (фирма Everprecision, Тайвань), предназначенного для гравирования прецизионных печатных плат. Точность позиционирования станка в горизонтальной плоскости составляет $5 \mu \mathrm{m}$, а по вертикали $9 \mu \mathrm{m}$, размер рабочей области составляет $300 \times 200 \times 20 \mathrm{~mm}$. Программное обеспечение станка позволяет в ручном режиме управлять перемещением с заданным шагом нажатием кнопок в окне программы. Для управления станком в автоматическом режиме разработано интерфейсное приложение, которое отправляет сообщения программному обеспечению станка, имитирующие нажатия клавиш. Датчик магнитометра и предметный столик вынесены на прикрепленную к станку немагнитную консоль. Ее использование уменьшает искажение магнитного поля деталями станка, но приводит к появлению дополнительной погрешности при позиционировании преобразователя, которую необходимо компенсировать калибровкой, алгоритм которой описан ниже.

Программная часть реализует как алгоритм калибровки, так и алгоритм восстановления поверхностной плотности тока по измеренному на заданной вне образца поверхности распределению индукции магнитного поля. Для тонкой проводящей пленки можно считать, что вектор ј лежит в плоскости пленки, равномерно распределен по ее толщине и создает вне пленки магнитную индукцию [6,7]:

$$
\begin{gathered}
B_{x}(x, y)=\frac{\mu_{0} d}{4 \pi z^{2}(x, y)} \iint_{\Omega} K\left(\frac{x-x^{\prime}}{z(x, y)}, \frac{y-y^{\prime}}{z(x, y)}\right) j_{y}\left(x^{\prime}, y^{\prime}\right) d x^{\prime} d y^{\prime}, \\
B_{y}(x, y)=\frac{\mu_{0} d}{4 \pi z^{2}(x, y)} \iint_{\Omega} K\left(\frac{x-x^{\prime}}{z(x, y)}, \frac{y-y^{\prime}}{z(x, y)}\right) j_{x}\left(x^{\prime}, y^{\prime}\right) d x^{\prime} d y^{\prime},
\end{gathered}
$$

Письма в ЖТФ, 2017, том 43, вып. 15 


$$
\begin{aligned}
B_{z}(x, y) & =\frac{\mu_{0} d}{4 \pi z^{3}(x, y)} \iint_{\Omega} K\left(\frac{x-x^{\prime}}{z(x, y)}, \frac{y-y^{\prime}}{z(x, y)}\right) \\
& \times\left(j_{x}\left(x^{\prime}, y^{\prime}\right)\left(y-y^{\prime}\right)-j_{y}\left(x^{\prime}, y^{\prime}\right)\left(x-x^{\prime}\right)\right) d x^{\prime} d y^{\prime} .
\end{aligned}
$$

Здесь

$$
K(u, v)=\left(1+u^{2}+v^{2}\right)^{-3 / 2}
$$

- интегральное ядро задачи (1), ширина которого порядка высоты $z$; $d$ - малая толщина образца; $\Omega$ - поверхность образца, совпадающая с плоскостью $O x y ; z(x, y)$ - расстояние от точки измерения магнитной индукции до поверхности образца.

Спектральные методы решения уравнений (1) обеспечивают пространственное разрешение, которое определяется шириной интегрального ядра (2) на полувысоте [7]. Получить значительно лучшее разрешение можно при наличии априорной информация о токовом распределении, которая учитывается в его параметрической модели. В качестве модели образца использовался ленточный диполь (рис. 1) с параметрами: ток $I$, координата центра диполя $x_{0}$, ширина полос $w$, расстояние между центрами полос $l$, их толщина $d(d \ll z(x, y)$ и $d \ll l)$. Тогда из формулы (1) в системе координат образца получаем

$$
\begin{aligned}
B_{x}^{m}(x, y, z(x, y))= & F_{2}(x, y, z,-w-l)-F_{2}(x, y, z,+w-l) \\
- & F_{2}(x, y, z,-w+l)+F_{2}(x, y, z,+w+l), \\
& B_{y}^{m}(x, y, z(x, y))=0 ; \\
B_{z}^{m}(x, y, z(x, y))= & F_{1}(x, y, z,-w-l)-F_{1}(x, y, z,+w-l) \\
- & F_{1}(x, y, z,-w+l)+F_{1}(x, y, z,+w+l),
\end{aligned}
$$

где

$$
F_{1}(x, y, z, u)=\frac{\mu_{0} I}{2 \pi w} \ln \left(z^{2}(x, y)+\left(\left(x-x_{0}\right)+u / 2\right)^{2}\right)
$$

и

$$
F_{2}(x, y, z, u)=\frac{\mu_{0} I}{2 \pi w} \operatorname{arctg}\left(\frac{2\left(x-x_{0}\right)+u}{z(x, y)}\right) .
$$

Калибровка микроскопа проводится по изготовленному станком ЕР2006Н на фольгированном текстолите и закрепленном на плоском предметном столике ленточном диполе с $w=0.3 \mathrm{~mm}, l=0.4 \mathrm{~mm}$, 


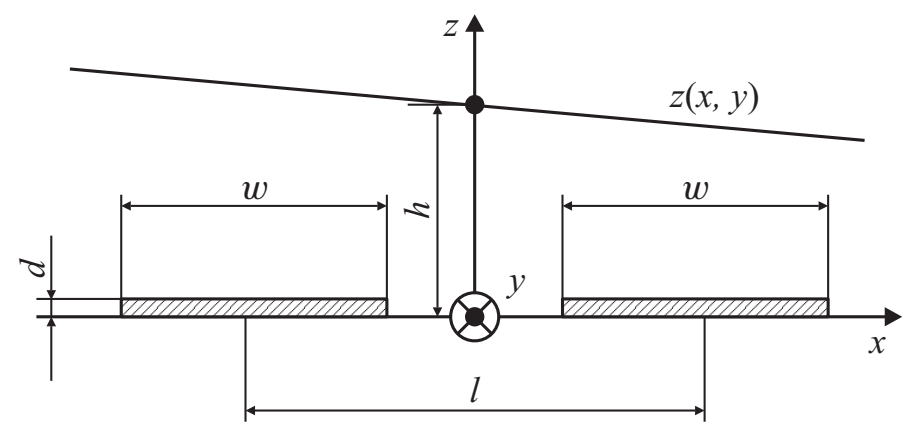

Рис. 1. Сечение ленточного диполя.

$d=56 \mu \mathrm{m}$, который задает систему координат образца $O x y z$, ленты располагаются параллельно оси $y$. Калибровка заключается в определении ориентации чувствительной оси датчиков Холла $\left\{n_{x}, n_{y}, n_{z}\right\}$, коэффициента его преобразования $S$, смещения начала координат $\{0,0, h\}$ системы координат измерителя $O^{\prime} x^{\prime} y^{\prime} z^{\prime}$ в системе координат образца $O x y z$ и углов Эйлера прецессии $\alpha$, нутации $\beta$ и собственного вращения $\gamma$ поворота системы $O^{\prime} x^{\prime} y^{\prime} z^{\prime}$ относительно $O x y z$ [8]. Уравнение плоскости, в которой перемещается датчик магнитометра, в системе координат образца имеет вид: $z(x, y)=\sin (\alpha) \operatorname{tg}(\beta) x-\cos (\alpha) \operatorname{tg}(\beta) y+h$ и используется в соотношении (3). Вектор $\widetilde{\mathbf{B}}$ индукции магнитного поля ленточного диполя (3) в системе координат измерителя $O^{\prime} x^{\prime} y^{\prime} z^{\prime}$ имеет вид

$$
\left(\begin{array}{c}
\tilde{B}_{x} \\
\tilde{B}_{y} \\
\tilde{B}_{z}
\end{array}\right)=\left(\begin{array}{ccc}
\cos \alpha \cos \gamma-\sin \alpha \cos \beta \sin \gamma & -\cos \alpha \sin \gamma-\sin \alpha \cos \beta \cos \gamma & \sin \alpha \sin \beta \\
\sin \alpha \cos \gamma+\cos \alpha \cos \beta \sin \gamma & -\sin \alpha \sin \gamma+\cos \alpha \cos \beta \cos \gamma & -\cos \alpha \sin \beta \\
\sin \beta \sin \gamma & \sin \beta \cos \gamma & \cos \beta
\end{array}\right)\left(\begin{array}{c}
B_{x}^{m} \\
0 \\
B_{z}^{m}
\end{array}\right) .
$$

В процессе калибровки с шагом $\Delta X$ и $\Delta Y$ измеряются распределения отсчетов напряжений Холла над калибровочным диполем $U^{+}[n, k]$ при прямом и $U^{-}[n, k]$ обратном направлении тока через диполь, где $U^{ \pm}[n, k]=U^{ \pm}(n \Delta X, k \Delta Y)$. Для исключения постоянной составляющей магнитного поля Земли и постоянного смещения холловского магнитометра вычисляется полуразность $U[n, k]=\left(U^{+}[n, k]-U^{-}[n, k]\right) / 2$. Затем с помощью генетического алгоритма [9] подбираются параметры $\alpha, \beta$,

Письма в ЖТФ, 2017, том 43, вып. 15 
$\gamma, h, n_{x}, n_{y}, n_{z}, S$ таким образом, чтобы минимизировать сумму

$$
\begin{aligned}
& J\left(\alpha, \beta, y, n_{x}, n_{y}, n_{z}, S, I, x_{0}\right)=\sum_{n, k} \mid U[n, k] \\
& -S\left(n_{x} \tilde{B}_{x}[n, k]+n_{y} \tilde{B}_{y}[n, k]+n_{z} \tilde{B}_{z}[n, k]\right) \mid,
\end{aligned}
$$

где зависимость компонент вектора $\tilde{\mathbf{B}}[n, k]$ от параметров определена формулами (3) и (4); суммирование производится по всем точкам распределения, а ток $I$ и координата диполя $x_{0}$ считаются известными. Применение генетического алгоритма обусловлено необходимостью найти глобальный экстремум нелинейного функционала ошибки (5), минимизация которого проводится в три этапа. На первом этапе область поиска решения в пространстве параметров определялась из условий постановки эксперимента. Результатом первого этапа является набор приближенных значений параметров, соответствующих локальным экстремумам функционала (5). На втором этапе новая область поиска решений выбирается минимальной, охватывающей весь полученный на первом этапе набор; если ее объем в пространстве параметров существенно меньше, чем на предыдущем этапе, поиск повторяется в новой области. На третьем этапе используется алгоритм градиентного спуска для получения точного решения. После калибровки магнитного микроскопа ток калибровочного диполя отключался.

Для определения метрологических характеристик микроскопа калибровка выполнялась несколько раз при токе через калибровочный диполь $104 \mathrm{~mA}$. Сканирование проводилось на участке $5 \times 1 \mathrm{~mm} \mathrm{c}$ шагом по $0.2 \mathrm{~mm}$. Погрешность определения высоты $h$ составила меньше $20 \mu \mathrm{m}$, погрешность ориентации датчика Холла и углов Эйлера не превосходит $10^{-3} \mathrm{rad}$, относительная погрешность определения крутизны преобразования датчика Холла не превосходит $10^{-3}$.

Исследуемое токовое распределение задавалось ленточным диполем, идентичным калибровочному и смещенным относительно него на расстояние $x_{0}=3.500 \mathrm{~mm}$. Измерялось распределение магнитного поля и производилась минимизация функционала (5) с учетом соотношений (3) и (4) по параметрам $\left(x_{0}, I\right)$. Остальные параметры задачи были определены на этапе калибровки. Относительная погрешность определения тока $I$ через диполь не превышает $10^{-4}$ и находится на уровне стабильности источника тока. Измеренное распределение 


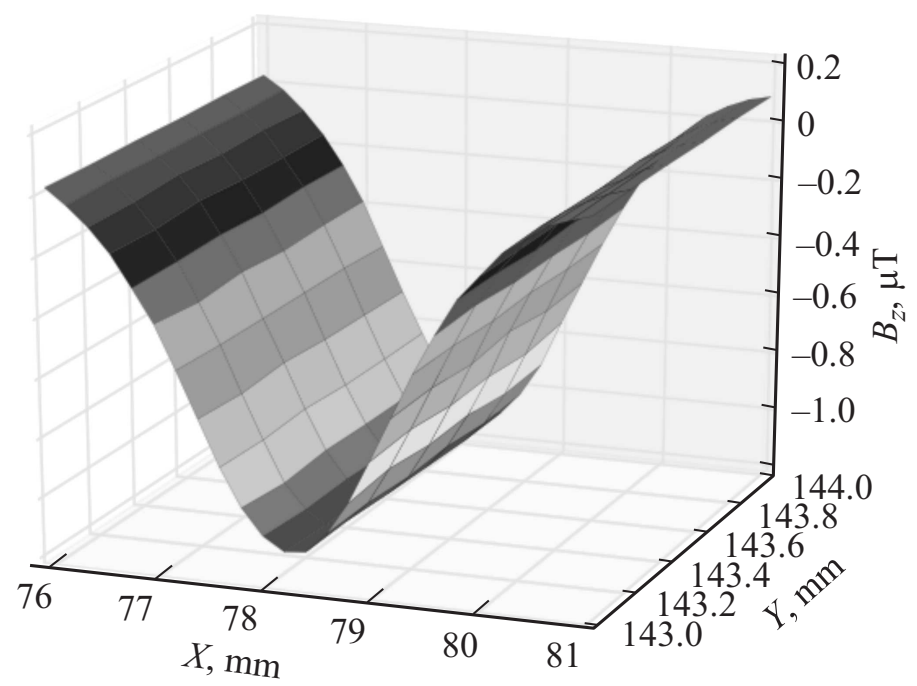

Рис. 2. Нормальная компонента индукции магнитного поля, создаваемого ленточным диполем.

магнитного поля над ленточным диполем при $h=3 \mathrm{~mm}$ приведено на рис. 2. Пространственное разрешение составляет около $3 \mathrm{~mm}$ и соответствует расстоянию от датчика до объекта.

В результате минимизации функционала (5) ошибка в определении координаты измеряемого диполя составила около $2 \mu \mathrm{m}$, что существенно меньше не только расстояния между датчиком микроскопа и поверхностью образца, но и размеров активной зоны используемого преобразователя. Полученная ошибка приближается к рассчитанному для ленточного диполя пределу Рао-Крамера [10], составляющему около $1.9 \mu \mathrm{m}$, что говорит об эффективности используемого в магнитном микроскопе метода обработки данных.

Исследования показали, что основной вклад в погрешность позиционирования вносит систематическая составляющая, которая устраняется при калибровке. Случайная составляющая погрешности позиционирования после калибровки составляет $1-2 \mu \mathrm{m}$, что соответствует паспортным характеристикам станка ЕР2006Н, и именно

Письма в ЖТФ, 2017, том 43, вып. 15 
она определяет пространственное разрешение магнитного микроскопа. При уменьшении СКО отсчетов магнитометра до $1 \mathrm{nT}$ [5] предел Рао-Крамера [10] пространственного разрешения составит около $20 \mathrm{~nm}$. Для того чтобы достигнуть такого разрешения, необходима система микроперемещений с погрешностью не более $20 \mathrm{~nm}$, например на основе пьезоэлектрических актуаторов [11]. Большой размер активной зоны преобразователя Холла существенно не повлиял на результат определения пространственного положения измеряемого диполя, следовательно, для данной задачи использование микродатчиков с меньшей чувствительностью и большими тепловыми шумами нецелесообразно.

Измерительный магнитный микроскоп может определять параметры любого токового распределения, например кругового токового диполя, допускающего интегрирование выражений (1). Плотность тока $\mathbf{j}$ в уравнениях (1) может быть заменена намагниченностью по формуле $\mathbf{j}=-\operatorname{rot}(\mathbf{M})$. При таком подходе, можно определять параметры распределения нормальной компоненты намагниченности тонких пленок с погрешностью значительно меньшей, чем достижимо спектральным методом. Это позволит оценивать распределение механических напряжений в намагниченных материалах по топографии магнитного поля вблизи их поверхности.

Работа выполнена за счет гранта Российского научного фонда № 15-19-00028.

\section{Список литературы}

[1] Oral A. et al. // Appl. Phys. Lett. 1996. V. 69 (9). P. 1324-1326.

[2] Ростами Х.Р. // Приборы и техника эксперимента. 2004. № 6. С. 95-102.

[3] Ростами X.P. // Приборы и техника эксперимента. 2016. № 2. С. 112-116.

[4] Игнатьев В.К., Перченко С.В. // Приборы и техника эксперимента. 2013. № 6. C. 117.

[5] Голубев А.А., Игнатьев В.К., Никитин А.В. // Приборы и техника эксперимента. 2008. № 5. С. 123-128.

[6] Bludova A.I. // Phys. Procedia. 2015. V. 71. P. 394-400.

[7] Likkason O.K. // Adv. Geosci. Remote Sens. 2014. P. 141-174.

[8] Берёзкин Е.Н. Курс теоретической механики. Изд. 2. М.: Изд-во МГУ, 1974. $641 \mathrm{c}$. 
[9] Tarek A. El-Mihoub, Adrian A. Hopgood, Lars Nolle, Alan Battersby // Eng. Lett. 2006. V. 13. N 2. P. 124-137.

[10] Kay S.M. Fundamentals of Statistical Signal Processing: Estimation Theory. Prentice Hall, Upper Sadle River, NJ, 1998. 595 p.

[11] Chi Z., Xu Q. // Int. J. Adv. Robot. Syst. 2014. V. 11. doi: 10.5772/59099

Письма в ЖТФ, 2017, том 43, вып. 15 